\title{
Big Data and Deep Learning Model for FMS Score Prediction of Aerobics Athletes
}

\author{
Wenying Xiong, Dongqin Huang, and Wei Xu $(D$ \\ College of Physical Education and Health, Jiangxi University of Traditional Chinese Medicine, Nanchang 330000, Jiangxi, China \\ Correspondence should be addressed to Wei Xu; 20112001@jxutcm.edu.cn
}

Received 20 April 2021; Revised 18 June 2021; Accepted 3 July 2021; Published 17 July 2021

Academic Editor: Shah Nazir

Copyright (c) 2021 Wenying Xiong et al. This is an open access article distributed under the Creative Commons Attribution License, which permits unrestricted use, distribution, and reproduction in any medium, provided the original work is properly cited.

In recent years, competitive aerobics has developed rapidly in my country, and the corresponding sports injury risks have gradually increased. A number of studies have shown that due to the characteristics of aerobics itself, difficult movement requirements, fast-paced music accompaniment and coherent coordinated movements, athletes will suffer sports injuries if they are not paying attention. Therefore, discovering the causes of athletes' injuries in time and preventing them in time is crucial for improving athletes' skill level and prolonging sports life. Through the functional movement screening (FMS) test, understanding young aerobics athletes' insufficiency in trunk stability, joint flexibility, muscle extension, and core strength can further help athletes reduce the risk of sports injuries. Therefore, this article proposes a novel sports injury risk model based on big data technology and deep learning, which can effectively predict the risk of sports injury and can play a positive role in improving the quality of athletes' movements and prolonging their sports life.

\section{Introduction}

In recent years, competitive aerobics [1-3] has developed rapidly in my country, and the corresponding sports injury risks [4-6] have gradually increased. A number of studies have shown that due to the characteristics of aerobics itself, strict time requirements, more difficult action requirements, fast-paced music accompaniment, and coherent coordinated actions, athletes will suffer sports injuries if they are not paying attention. The shoulders, elbows, wrists, waists, thighs, knees, calves, and ankles are the most prone to injury during aerobics training [7-9]. Among them, the most prone to injury is the ankle joint. In addition, the type of injury most likely to occur for competitive aerobics athletes is closed injury, most of which are joint strain, sprain and muscle strain, and chronic injuries are the main ones. However, the current scholars' research on aerobics injuries is usually carried out through manual investigation methods.

Aerobics athletes are in the golden stage of physical development [10]. During this period, various physical qualities will be significantly improved. However, in interviews with aerobics athletes and coaches, it is found that the types of injuries of aerobics athletes are higher than those of other athletes. Moreover, this is due to the weakness of the aerobics athletes' own muscles and joints, which limits their skill development ability [11] and long-term training of irregular technical movements and body postures. In the teenage years of aerobics athletes, scientific and reasonable training can not only promote the physical development of adolescents but also improve their athletic ability more effectively. Therefore, discovering the causes of aerobics athletes' injuries in time and preventing them in time is crucial for improving athletes' skill level and prolonging sports life [12].

Functional movement screening [13-15] is a test model proposed by American orthopedic training experts in 1996. The test mainly uses 7 functional movements to detect the stability of the subjects' overall movement, joint flexibility, softness, balance ability, core strength, and proprioception, find out whether the subject's movement pattern exists or is potentially compensatory and noncompetitive symmetry, and then analyze the risk of subjects' sports injuries. As long 
as there is a serious asymmetry or defect in the action posture, it is necessary to use accurate and appropriate corrective training to correct the wrong posture in time and, at the same time, make more effective suggestions so that the athlete can better prevent the risk of injury. For more than ten years, the FMS test has been continuously applied and adjusted in functional sports and clinical aspects. The test methods and scoring rules have been very complete and standardized. At present, as an important supplement to traditional training methods, FMS has been widely used in professional sports leagues.

At present, domestic FMS research has a wide range of research objects in the field of sports, but there are few research studies on young aerobics athletes. Young athletes are related to the development of sports in the future, and their physical ability is as important as their sports life span. Therefore, through the FMS test, understanding young aerobics athletes' insufficiency in trunk stability, joint flexibility, muscle extension, and core strength can further help athletes reduce the risk of sports injuries. Therefore, this paper constructs an FMS prediction algorithm based on big data technology and deep learning [16-20], which can further improve the prediction accuracy of motion loss risk. The following are the main innovative points of this paper:

(i) A novel sports injury risk model based on big data technology and deep learning, which can effectively predict the risk of sports injury and can play a positive role in improving the quality of athletes' movements and prolonging sports life

(ii) FMS test and deep neural network to construct a joint algorithm for sports injury prediction, which can further improve the accuracy of sports injury risk prediction

(iii) Simulation and ablation experiments are carried out, and the experimental results prove the effectiveness and superiority of the algorithm in this paper

\section{Related Work}

2.1. FMS. FMS, functional motor screening, was first proposed by American orthopedic training experts Gray Cook and Lee Burton. Derived from the famous functional movement training, it was first applied in the 1990s. At present, it has been widely used as a testing method in the field of physical therapy, rehabilitation, and physical training and is suitable for all kinds of people. FMS test combines the comprehensive knowledge of sports anatomy, sports physiology, sports biomechanics, and neurology to connect with the basic movements of the human body. After long-term practice and research, seven basic movements' tests and three exclusion tests are finally determined. From the standpoint of human basic sports ability, a comprehensive assessment of the human body is conducted using a simple scientific and intuitive method of grading basic action to detect human body movement coordination, flexibility, stability, and symmetry, and screening out the compensatory actions that are not conducive to the development of the level of movement and harm the body [21].

The test significance of FMS is to evaluate the quality of actions and to score and rank some of the defects and asymmetries of certain action patterns so that specialized technical actions can be better developed. As a result, Cook proposed the best performance pyramid (Figure 1) and pointed out that the first layer is "Movement": the human body's most basic athletic ability, that is, the most fundamental flexibility and stability in sports. The second layer is "Performance": the speed, strength, endurance, and other qualities of human movement. The third layer is "Skill." Cook puts forward that the flexibility of the body and the ability to control stability are the basis for the free movement of the human body during exercise, and it also provides a guarantee for the completion of highquality movements so as to achieve a higher level of exercise goals.

Lloyd et al. [22] found in the study of juvenile football players that the total FMS score will also be affected by the age of the player and the level of different athletic abilities displayed. The "physical maturity" shown by athletes of different ages is closely related to the quality of movement completion in the FMS test. Marques et al. [23] conducted FMS tests on young football players aged 14-20 and found that young football players have asymmetrical heights on the left and right sides of the body. $91 \%$ of the players had 0 or 1 points during the test. $82 \%$ of the athletes' test score does not exceed 14 points, indicating that they have a higher potential risk of injury.

2.2. Sports Injuries of Aerobics. Malliou et al. [24] used a questionnaire survey method and conducted statistics and analysis on the injuries of athletes engaged in aerobics training and found that, in the injured population, lower extremity injuries accounted for $97.3 \%$, and the injuries of the stomp joints and knee joints are the most common in aerobics training. At the same time, it is pointed out that the training time, years, and training level will all have an impact on the injury. Bintoudi et al. [25] investigated two aerobics pedal athletes and found that they had knee joint pain and fat pad edema. They also discussed the possible pathogenic factors and mechanisms of aerobics.

\section{Methodology}

Due to the characteristics of competitive aerobics, athletes are required to complete a series of high-intensity movements in a short time, which requires a higher level of physical fitness and physical flexibility of the athletes. Studies have confirmed that long-term high-intensity repetitive exercise training and asymmetric sports skills and postures will increase the risk of athletes' injuries. At the same time, taking into account the rapid development of big data and neural network technology [26-30], the construction of aerobics sports injury is feasible. 


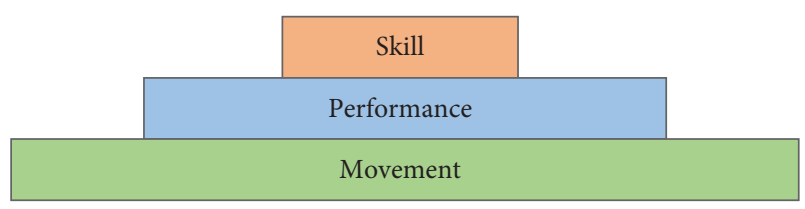

Figure 1: Best performance pyramid.

3.1. The Functional Movement System of Aerobics Athletes. FMS motion screening detects weak points in human movement to correct incorrect posture and reduces the risk of sports injury. The functional movement system (FMS) includes seven test movements, including squat over the top mode, hurdle step, lunge squat, shoulder flexibility, straight leg lift, push-ups, and rotational stability. The functional movement screening test only detects pain or no pain and does not assign a score. A total of 7 functional movements are scored on a scale of 0 to 3, with a total of four scores based on how well the subjects complete the above 7 basic test movements. The subjects will get 3 points if they complete the specified action pattern, with the highest score of 3 points; 2 points will be given to the subjects who are in the mode of completion (having the ability to complete) but have compensatory actions or poor quality of completion when completing the actions; 1 point will be given when the subject cannot complete the functional movements or does not have the ability to complete the movements and fails to maintain body balance during the movements. The injured person has pain in any part of the body at the time of completing the action. A total FMS score of 21, with a functional screening score of less than 14 indicating a greater risk of injury during training, is obtained; the higher the score, the lower the risk of injury.

\subsection{Sports Injury Prediction Model}

3.2.1. Analysis of Time and Space Correlation of Aerobics. We know that aerobics actions have time and space correlation (as shown in Figure 2), so this article uses the CNN model [31-33] to extract the time and space characteristics of aerobics actions and effectively utilizes the time and space characteristics of aerobics actions. First, we introduce the theory of correlation analysis.

(1) Covariance: in traditional statistical analysis, covariance can measure the joint change of two random variables. The sign of covariance reflects the linear relationship between variables. Suppose the random variables are $X$ and $Y$; then, the covariance calculation equation between these two random variables is as follows:

$$
\operatorname{cov}(X, Y)=E\{[X-E(X)][Y-E(Y)]\},
$$

where $E(X)$ and $E(Y)$ are the expected values of random variables $X$ and $Y$, respectively. Specifically, the covariance represents the expectation of the overall error of the two variables.

(2) Pearson correlation coefficient: the correlation coefficient was put forward by the statistician Karl
Pearson. It is an index to analyze the degree of linear correlation among variables. The Pearson correlation coefficient is widely used. Assuming that there are two sets of data sets $X$ and $Y, n$ is the sample size, and $r$ is used to represent the Poisson correlation coefficient of the two. The calculation equation of the Poisson correlation coefficient is as follows:

$$
r=\frac{\operatorname{cov}(X, Y)}{\sqrt{\sigma_{X}^{2}} \sqrt{\sigma_{Y}^{2}}}=\frac{\sum_{i=1}^{n}\left(x_{i}-\bar{x}\right)\left(y_{i}-\bar{y}\right)}{\sqrt{\sum_{i=1}^{n}\left(x_{i}-\bar{x}\right)^{2}} \sqrt{\sum_{i=1}^{n}\left(y_{i}-\bar{y}\right)^{2}}},
$$

where $\operatorname{cov}(X, Y)$ is the covariance of $X$ and $Y, \sigma_{X}^{2}$ and $\sigma_{Y}^{2}$ are the variances of the variables $X$ and $Y$, respectively, and $\overline{\mathrm{x}}$ and $\overline{\mathrm{y}}$ are the mean values of the variables $x$ and $y$.

By extending the low-dimensional random vector to the high-dimensional random vector, the correlation coefficient matrix can be obtained, and each element in the correlation coefficient matrix is the correlation coefficient of the row vector and column vector where it is located. Assuming $\theta$ is a column vector composed of $n$ scalars randomly, $\theta=\left[X_{1}, X_{2}, \ldots, X_{n}\right]$, the calculation equation of the correlation coefficient matrix is as follows:

$$
r=\left[\begin{array}{cccc}
r_{11} & r_{12} & \cdots & r_{1 n} \\
r_{21} & r_{22} & \cdots & r_{2 n} \\
\vdots & \vdots & \ddots & \vdots \\
r_{n 1} & r_{n 2} & \cdots & r_{n n}
\end{array}\right] .
$$

The calculation equation for finding the element value of the $i$ th row and $j$ th column of the correlation coefficient matrix $r$ is as follows:

$$
r_{i j}=\frac{\operatorname{cov}\left(x_{i}, x_{j}\right)}{\sigma_{x_{i}} \sigma_{x_{j}}}=\frac{E\left\{\left[X_{i}-E\left(X_{i}\right)\right]\left[X_{j}-E\left(X_{j}\right)\right]\right\}}{\sqrt{E\left(X_{i}^{2}\right)-E^{2}\left(X_{i}\right)} \sqrt{E\left(X_{j}^{2}\right)-E^{2}\left(X_{j}\right)}},
$$

where $\sigma_{X_{i}}$ and $\sigma_{X_{j}}$ are the mean square deviations of random variables $X_{i}$ and $X_{j}$, respectively.

\subsubsection{Construction of Spatiotemporal Feature Matrix.} The following is the specific approach for the original data refactoring and data integration for the matrix form as a model of the original input data in this paper: to begin, combine the adjacent section 25 testing points with 15 consecutive time nodes of aerobics movement data, with a 5-second interval between time nodes, and then build each testing point of time series data as given in the equation below:

$$
P_{i, j}=\left[t_{i, 1}, t_{i, 2}, \ldots, t_{i, 15}\right],
$$

where $t_{i, j}$ represents the $i$ th detection point and the aerobics action data of the $j$ th time node among the selected 15 time nodes. Finally, the data of each detection point is integrated, and the calculation equation of the $15 \times 15$ space-time characteristic matrix is as follows: 


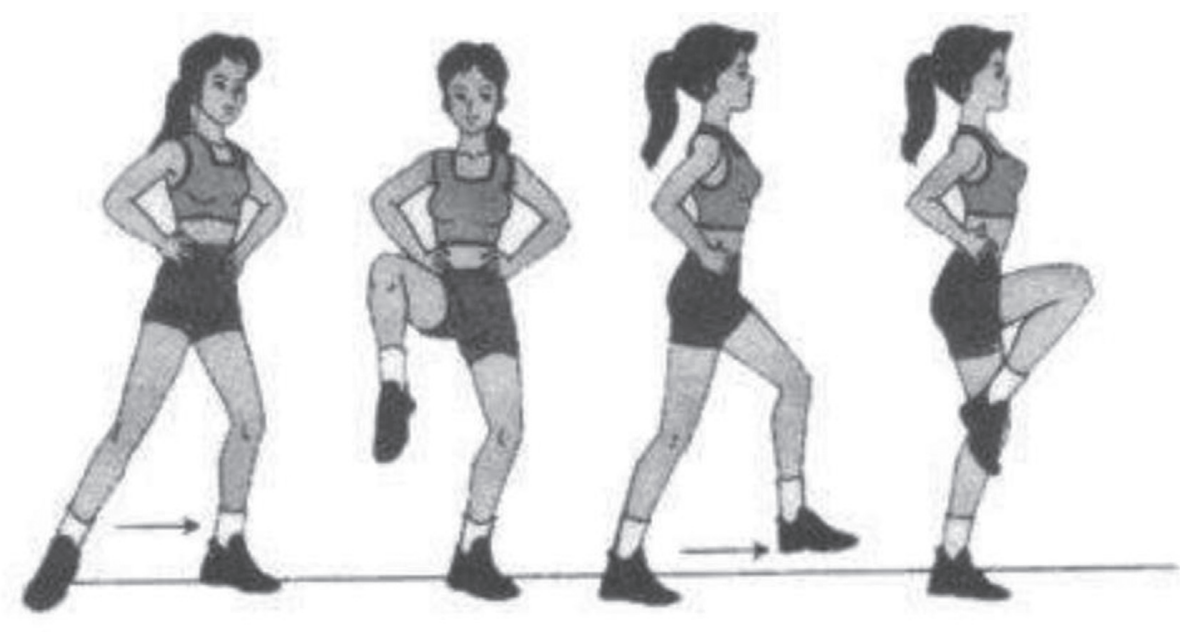

FigURE 2: Basic structure of standard recurrent neural network.

$$
D=\left[\begin{array}{cccc}
t_{1,1} & t_{1,2} & \cdots & t_{1,15} \\
t_{2,1} & t_{2,2} & \cdots & t_{2,15} \\
\vdots & \vdots & \ddots & \vdots \\
t_{15,1} & t_{15,2} & \cdots & t_{15,15}
\end{array}\right] .
$$

The matrix form of aerobics action data constructed above fully considers the spatiotemporal correlation, but the amount of data is still too large, resulting in time-consuming model training and increased complexity. In response to this problem, we use convolutional neural networks to simplify matrix features. Convolution filtering and downsampling are two operations to extract features.

3.2.3. CNN Model. Multilayer CNN includes convolution layer, pooling layer, full connection layer, and output layer. Neurons in adjacent layers are connected to each other, but neurons in the same layer are not connected to each other. We divide CNN into three parts: input feature matrix, feature extraction, and feature vector output. Feature extraction mainly includes convolution and pooling. The convolutional layer $C$ carries out convolution calculation through multiple convolutional check input features to obtain multiple convolutional feature vectors, and the pooling layer will carry out local feature extraction. The convolution calculation equation is as follows:

$$
X_{i, j}=f\left[\sum_{q-1}^{r} \sum_{p-1}^{r}\left(D_{(i+p)(j+q)} C_{p q}\right)+b_{c}\right] .
$$

The constructed feature matrix uses the rxr convolution kernel $c$ to perform a sliding calculation with a step length of 1 plus the bias variable $b$ to obtain a feature vector of $(n-r+1) x(m-r+1)$ dimensions. Input the feature vector into the activation function factory. The calculation equation of the linear rectification ( $\mathrm{ReLU}$ ) activation function used in this paper is as follows:

$$
f(x)=\max (0, x) .
$$

Compared with activation functions such as sigmoid, this function reduces the computational complexity, overcomes the problem of gradient disappearance, and converges faster. The convolution calculation uses partially connected features to simplify some features of the feature matrix. During iteration, the principle of gradient descent is used to continuously adjust the shared weights in the convolution kernel so that the data features can be fundamentally mined.

3.2.4. CNN Extracts Spatiotemporal Features. We use the preprocessed aerobics action data of 15 adjacent detection points, and the data of the first 125 seconds of a single sample of each detection point constructs a $15 \times 15$ feature matrix. We set two convolutions and two pooling processing feature matrices, and the ReLU activation function is introduced. The size of the first layer of convolution kernel is set to $3 \mathrm{X} 3$, the step size is set to 1 , and the size of the feature matrix obtained after one convolution is $23 \times 23$; then, the average pooling is performed, the size is set to $2 \times 2$, and the step size is set to 2; here, padding is set to "SAME," and the size of the feature matrix after one pooling becomes $12 \times 12$; then, after another convolution, the size of the convolution kernel is set to $3 \times 3$, and the step size is set to 1 , and we get the $10 \times 10$ feature matrix which is subjected to mean pooling again. The size is set to $2 \times 2$, the step size is 2 , and the $5 \times 5$ feature matrix is obtained. The CNN model structure is shown in Figure 3.

\section{Experiments and Results}

4.1. Experimental Subject. In this study, 200 college students from a certain city were selected as the research objects. According to gender and aerobics level, they were divided into male aerobics group, male first-grade aerobics group, male second-grade aerobics group, male general student group, and female athlete group. There was no statistical difference in basic information such as height, weight, and $\mathrm{BMO}$ among 10 people in each group, including the firstlevel female aerobics group, the second-level female aerobics 


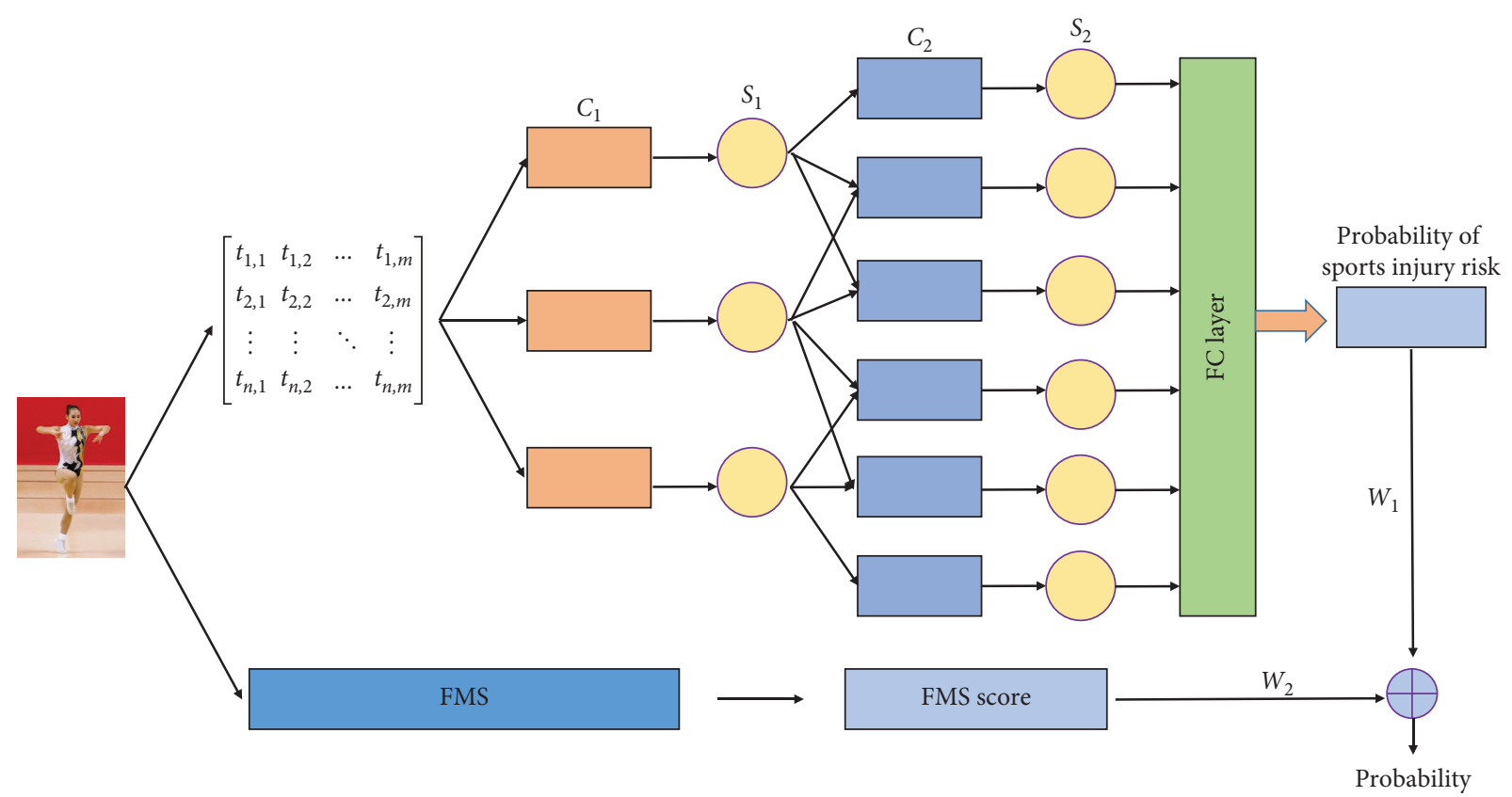

Figure 3: The overall structure of our model.

TABLE 1: Basic situation of the research object.

\begin{tabular}{|c|c|c|c|c|c|}
\hline Group & Sample size & Age & Height $(\mathrm{cm})$ & Weight $(\mathrm{kg})$ & BMI \\
\hline Master aerobics group & 10 & 21.5 & 172.1 & 62.3 & 21.1 \\
\hline Level 1 aerobics group & 10 & 22.1 & 174.3 & 63.1 & 20.5 \\
\hline Level 2 aerobics group & 10 & 19.6 & 171.6 & 60.3 & 19.6 \\
\hline Ordinary student group & 10 & 18.9 & 168.8 & 58.3 & 21.4 \\
\hline
\end{tabular}

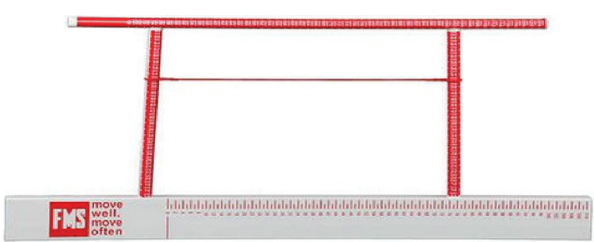

(a)

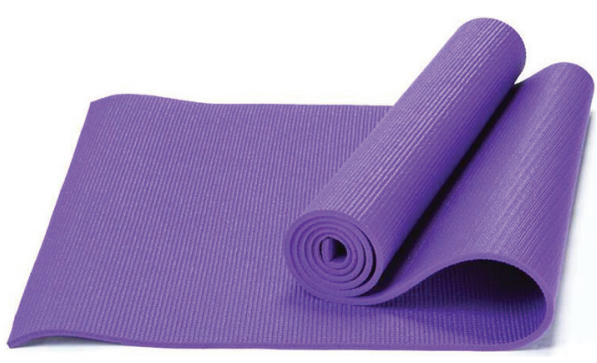

(b)

FIgURE 4: FMS test suite. (a) FMS suite. (b) Yoga mat.

TABLE 2: Experimental hardware platform and software simulation environment.

\begin{tabular}{lc}
\hline $\mathrm{CPU}$ & Intel (R) core (TM) i5-4200M CPU @ 2.50 GHz \\
\hline RAM & $8.00 \mathrm{~GB}$ \\
Operating system & Windows10 \\
Development environment & VS2015 \\
Development tools & OpenCV+Pycharm \\
\hline
\end{tabular}

group, and the female general student group. The basic situation is detailed in Table 1 , and the FMS test suite is shown in Figure 4.
4.2. Simulation Experiment Environment. The hardware environment and software environment are shown in Table 2. 


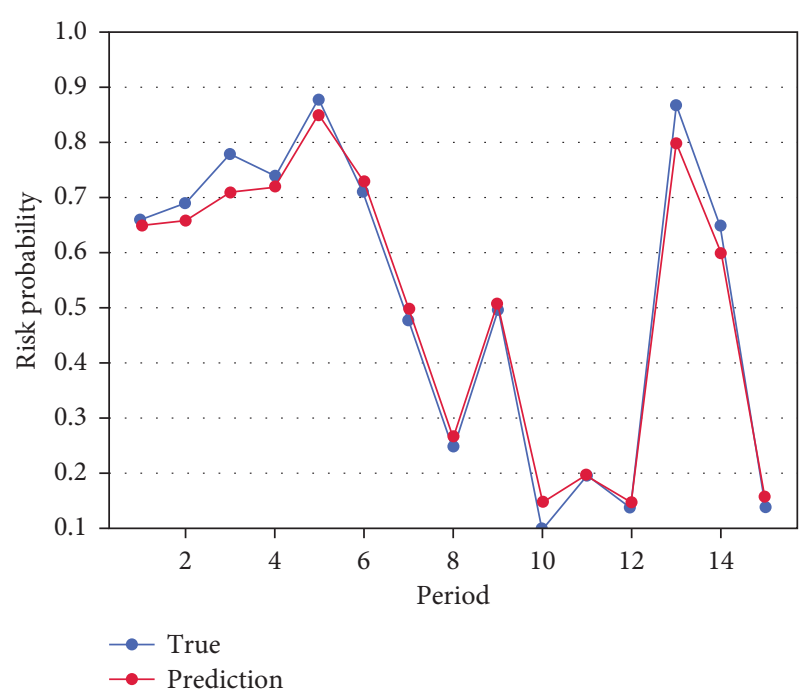

FIGURE 5: The prediction result of aerobics injury risk.

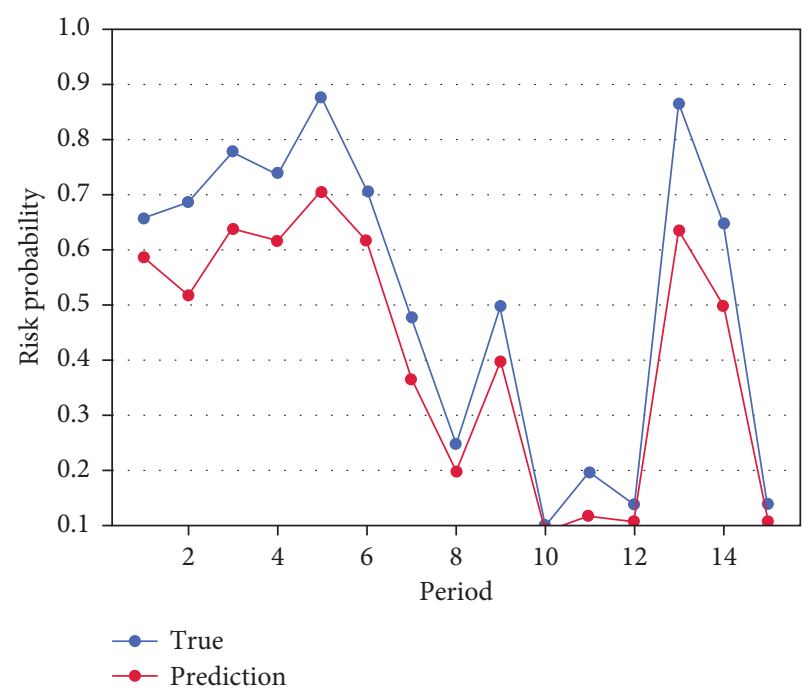

FIGURE 6: Prediction results of aerobics injury risk without FMS.

4.3. Experimental Results. In this section, experimental results are presented to verify the proposed system. From Figure 5, we can see that the new sports injury risk model proposed in this article based on big data technology and deep learning has achieved excellent performance and accurately predicts the possibility of aerobics sports injury risk. As can be seen from the figure, the prediction curve and the real curve are basically covered, which fully proves the effectiveness of the algorithm in this paper. In order to further demonstrate the advantages of the model in this paper, we also conducted ablation experiments to observe the effect of FMS on the performance of the model.

It can be clearly seen from Figure 6 that if FMS is not used, the prediction accuracy of aerobics injury risk will be greatly reduced. Therefore, this further proves the new sports injury risk model based on big data technology and deep learning proposed in this paper.

\section{Conclusion}

For the last few years, competitive aerobics have advanced speedily, and the corresponding sports injury risks have gradually increased. A number of studies have shown that due to the characteristics of aerobics itself, difficult movement requirements, fast-paced music accompaniment, and coherent coordinated movements, athletes will suffer sports injuries if they are not paying attention. As due to the rapid development of competitive aerobics, the risk of corresponding sports injuries has gradually increased. In addition, because of the timely detection of the cause of the athlete's injury and timely prevention, it is very important to improve the athlete's technical level and prolong the sports life. Therefore, this paper proposes a new type of sports injury risk model based on big data technology and deep learning. This model can effectively predict the risk of sports injury and has a positive effect on improving the athlete's sports quality and prolonging sports life.

\section{Data Availability}

The data used to support the findings of this study are included within the article.

\section{Conflicts of Interest}

The authors declare that they have no conflicts of interest.

\section{References}

[1] T. Moshenska and I. Bodrenkova, "Structure and content of competitive group compositions in sports aerobics," Slobozhanskyi Herald of Science and Sport, vol. 1, no. 57, pp. 40-42, 2017.

[2] S. Yue, "Image recognition of competitive aerobics movements based on embedded system and digital image processing," Microprocessors and Microsystems, vol. 82, Article ID 103925, 2021.

[3] Y. Peng, "Simulation algorithm of difficulty movements in competitive aerobics," in Proceedings of the International Conference on Cyber Security Intelligence and Analytics, pp. 614-617, Springer, Haikou, China, February 2020.

[4] H. Dhillon, S. Dhilllon, and M. S. Dhillon, "Current concepts in sports injury rehabilitation," Indian Journal of Orthopaedics, vol. 51, pp. 529-536, 2017.

[5] A. Hulme, J. Thompson, R. O. Nielsen, G. J. Read, and P. M. Salmon, "Towards a complex systems approach in sports injury research: simulating running-related injury development with agent-based modelling," British Journal of Sports Medicine, vol. 53, no. 9, pp. 560-569, 2019.

[6] International Olympic Committee Injury and Illness Epidemiology Consensus Group, R. Bahr, B. Clarsen et al., "International olympic committee consensus statement: methods for recording and reporting of epidemiological data on injury and illness in sports 2020 (including the STROBE extension for sports injury and illness surveillance (STROBE-SIIS))," Orthopaedic journal of sports medicine, vol. 8, no. 2, Article ID 2325967120902908, 2020.

[7] M. Behrens, K. Müller, J. I. Kilb et al., "Modified step aerobics training and neuromuscular function in osteoporotic patients: 
a randomized controlled pilot study," Archives of Orthopaedic and Trauma Surgery, vol. 137, no. 2, pp. 195-207, 2017.

[8] J. G. Zuzda, M. Pasek, R. Latosiewicz, and M. Bierkus, "Is there any effect of step aerobics training on physical fitness of female students?" in Proceedings of the International Conference of Computational Methods in Sciences and Engineering, vol. 2186, no. 1, December 2019, Article ID 080012.

[9] B. Xie, X. Yan, X. Cai, and J. Li, "Effects of high-intensity interval training on aerobic capacity in cardiac patients: a systematic review with meta-analysis," BioMed Research International, vol. 2017, Article ID 5420840, 16 pages, 2017.

[10] V. Pasichnyk, M. Pityn, V. Melnyk, I. Karatnyk, A. Hakman, and Y. Galan, "Prerequisites for the physical development of preschool children for the realization of the tasks of physical education," Physical Activity Review, vol. 6, pp. 117-126, 2018.

[11] K. Kendellen and M. Camiré, "Examining the life skill development and transfer experiences of former high school athletes," International Journal of Sport and Exercise Psychology, vol. 15, no. 4, pp. 395-408, 2017.

[12] R. P. Troiano, E. A. J. Frongillo, J. Sobal, and D. A. Levotsky, "Movements symmetrization-an effective method of injury prevention, health strengthening and prolonged sport careers of athletes," Movement in Human Life and Health, vol. 20, p. 35, 2020.

[13] M. Yu, T. Quan, Q. Peng, Yu Xu, and L. Liu, "A model-based collaborate filtering algorithm based on stacked autoencoder," Neural Computing \& Applications, vol. 1, 2021.

[14] W. D. Chang, L. W. Chou, N. J. Chang, and S. Chen, "Comparison of functional movement screen, star excursion balance test, and physical fitness in junior athletes with different sports injury risk," BioMed Research International, vol. 2020, Article ID 8690540, 10 pages, 2020.

[15] R. Arghadeh, A. Letafatkar, and S. S. Shojaeddin, "Relationship between physical fitness and functional movement screening scores in active males: providing preventing model," Journal of Clinical Physiotherapy Research, vol. 1, no. 1, pp. 13-20, 2018.

[16] X. Ning, Ke Gong, W. Li, L. Zhang, B. Xiao, and S. Tian, "Feature refinement and filter network for person re-identification," IEEE Transactions on Circuits and Systems for Video Technology, 2020.

[17] W. Cai and Z. Wei, "PiiGAN: generative adversarial networks for pluralistic image inpainting," IEEE Access, vol. 8, pp. 48451-48463, 2020.

[18] X. Ning, P. Duan, W. Li, and S. Zhang, "Real-time 3D face alignment using an encoder-decoder network with an efficient deconvolution layer," IEEE Signal Processing Letters, vol. 27, pp. 1944-1948, 2020.

[19] X. Zhang, Y. Yang, Z. Li, X. Ning, Y. Qin, and W. Cai, “An improved encoder-decoder network based on strip pool method applied to segmentation of farmland vacancy field," Entropy, vol. 23, no. 4, p. 435, 2021.

[20] W. Cai and Z. Wei, "Remote sensing image classification based on a cross-attention mechanism and graph convolution," IEEE Geoscience and Remote Sensing Letters, vol. 42, 2020.

[21] Y. Xu, D. Zhan, L. Liu, H. Lv, L. Xu, and J. Du, "A privacypreserving cross-domain healthcare wearables recommendation algorithm based on domain-dependent and domainindependent feature fusion," IEEE Journal of Biomedical and Health Informatics, 2021.

[22] R. S. Lloyd, J. L. Oliver, J. M. Radnor, B. C. Rhodes, A. D. Faigenbaum, and G. D. Myer, "Relationships between functional movement screen scores, maturation and physical performance in young soccer players," Journal of Sports Sciences, vol. 33, no. 1, pp. 11-19, 2015.

[23] V. B. Marques, T. M. Medeiros, F. de Souza Stigger, F. Y. Nakamura, and B. M. Baroni, "The Functional Movement Screen (FMS) in elite young soccer players between 14 and 20 years: composite score, individual-test scores and asymmetries," International journal of sports physical therapy, vol. 12 , no. 6 , p. $977,2017$.

[24] P. Malliou, A. Gioftsidou, G. Pafis, A. Beneka, and G. Godolias, "Proprioceptive training (balance exercises) reduces lower extremity injuries in young soccer players," Journal of Back and Musculoskeletal Rehabilitation, vol. 17, no. 3-4, pp. 101-104, 2004.

[25] A. Bintoudi, M. Goumenakis, and A. Karantanas, "Suprapatellar fat pad inflammation in step aerobics athletes: MR imaging evaluation of two cases," Open Medicine, vol. 7, no. 6, pp. 813-816, 2012.

[26] X. Ning, Ke Gong, W. Li, and L. Zhang, "JWSAA: joint weak saliency and attention aware for person re-identification," Neurocomputing, vol. 453, 2020.

[27] W. Cai, B. Liu, Z. Wei, M. Li, and J. Kan, "TARDB-Net: tripleattention guided residual dense and BiLSTM networks for hyperspectral image classification," Multimedia Tools and Applications, vol. 80, no. 7, pp. 11291-11312, 2021.

[28] Y. Tong, L. Yu, S. Li, J. Liu, H. Qin, and W. Li, "Polynomial fitting algorithm based on neural network," ASP Transactions on Pattern Recognition and Intelligent Systems, vol. 1, no. 1, pp. 32-39, 2021.

[29] J. Zhang, X. Jin, J. Sun, J. Wang, and K. Li, "Dual model learning combined with multiple feature selection for accurate visual tracking," IEEE Access, vol. 7, pp. 43956-43969, 2019.

[30] H. Khan, M. A. Jan, M. Alam, and W. Dghais, "A channel borrowing approach for cluster-based hierarchical wireless sensor networks," Mobile Networks and Applications, vol. 24, no. 4, pp. 1306-1316, 2019.

[31] J. Zhang, Y. Liu, H. Liu, and J. Wang, "Learning local-global multiple correlation filters for robust visual tracking with kalman filter redetection," Sensors, vol. 21, no. 4, p. 1129, 2021.

[32] Y. Ding, X. Zhao, Z. Zhang, W. Cai, and N. Yang, "Multiscale graph sample and aggregate network with context-aware learning for hyperspectral image classification," IEEE Journal of Selected Topics in Applied Earth Observations and Remote Sensing, vol. 14, pp. 4561-4572, 2021.

[33] J. Zhang, W. Wang, C. Lu, J. Wang, and A. K. Sangaiah, "Lightweight deep network for traffic sign classification," Annals of Telecommunications, vol. 75, no. 7, pp. 369-379, 2020. 